\title{
Arbeitsüberforderung und - unzufriedenheit von Lehrpersonen in der Schweiz - Zusammenhänge mit Depressivität und somatischen Beschwerden 1
}

\section{Marina Delgrande Jordan, Emmanuel Kuntsche und Jacqueline Sidler}

Die vorliegende Arbeit beschreibt erstmalig anhand einer repräsentativen Stichprobe von Lehrpersonen der 5. bis 9. Klassen in der Schweiz die psychometrischen Qualitäten der Skalen Arbeitsüberforderung und-unzufriedenheit von Enzmann und Kleiber (1989) sowie Zusammenhänge mit Depressivität und somatischen Beschwerden. Mit Hilfe von Hauptkomponentenanalysen und multiplen Regressionsanalysen wurden die Antworten von 485 Lehrpersonen untersucht. In Übereinstimmung mit vorangegangener Forschung konnten Arbeitsüberforderung und -unzufriedenheit als separate Dimensionen identifiziert werden. Arbeitsüberforderung und Depressivität sind in der Romandie weiter verbreitet als in der Deutschschweiz. In Bezug auf Arbeitsunzufriedenheit und somatische Beschwerden konnten keine Unterschiede zwischen den Sprachregionen festgestellt werden. Über ein Drittel der Gesamtvarianz von Depressivität konnte allein durch Arbeitsüberforderung und Arbeitsunzufriedenheit erklärt werden. Darüber hinaus gingen diese beiden Aspekte mit somatischen Beschwerden einher. Je überforderter und unzufriedener Lehrpersonen waren, desto mehr Beschwerden gaben sie an. Dies ist insofern Besorgnis erregend, als somatische oder psychische Beschwerden bei überforderten Lehrpersonen ein Indikator für erlebte Beanspruchungsfolgen wie z.B. chronischen Stress oder Burnout sein können. Die beschriebenen präventiven Massnahmen können dazu beitragen, die Arbeitsbedingungen der Lehrpersonen und das Arbeitsumfeld an den Schulen zu verbessern.

\section{Einleitung}

Zahlreiche epidemiologische Studien haben gezeigt, dass der Lehrberuf in besonderem Masse mit psychischen Belastungen einher geht und dass Lehrpersonen vor allem in der psychischen Gesundheit gefährdet sind. Es besteht international ein Konsens darüber, dass Lehrerinnen und Lehrer in diesem Zusammenhang als Risikopopulation betrachtet werden müssen (zusammenfassend: Schaarschmidt \& Fischer, 2001; Freitag, 1998; Rudow, 1994). 
Gemäss dem Rahmenmodell der Belastung und Beanspruchung von Rudow (1994) bestimmen die Arbeitsaufgaben der Lehrpersonen und die Bedingungen, unter denen sie erfüllt werden, die objektiven Belastungsfaktoren, welche unabhängig von der Lehrperson existieren und potenziell Beanspruchungen hervorrufen. Diese objektiven Belastungsfaktoren werden individuell kognitiv widergespiegelt und treten auf diese Weise als psychische Belastungen auf. In der Lehrtätigkeit treten gleichzeitig und dauerhaft zahlreiche verschiedenartige subjektive Belastungen auf, wie z.B. quantitative und qualitative Arbeitsüberforderung, fehlende Zeit für Vorbereitung und Durchführung des Unterrichts, Lärm, zu grosse Klassen oder Disziplinprobleme. Die effektive subjektive Belastung durch den Lehrberuf ist also durch das Zusammenwirken von mehreren Belastungsquellen bestimmt. Zur Frage, ob und inwieweit der Lehrberuf belastend sei, liegen zahlreiche empirische Studien vor, die eine hohe bis sehr hohe psychische Belastung bei Lehrpersonen zeigen (Bachmann, 1999; Rudow, 1994; Jarvis, 2002). Auch in der Schweiz wurde die psychische Belastung bei Lehrpersonen untersucht. In einer Studie, die in den Kantonen Freiburg und Luzern durchgeführt wurde, bezeichneten 35\% der Lehrpersonen ihre Arbeitsbelastung, z.B. durch Arbeitsüberforderung oder demotivierte Schulkinder, als hoch oder sehr hoch (Kramis-Aebischer, 1996). In einer Untersuchung im Kanton BaselStadt gaben 29\% der Lehrpersonen an, sich durch Arbeitsüberforderung stark bis sehr stark belastet zu fühlen (Ulich, Inversini, \& Wülser, 2002). Ähnliche Angaben lassen sich auch für die Kantone Luzern, Thurgau und Zürich finden (Frei, 1996). Zur Belastung trugen in der letztgenannten Studie vor allem die multiplen Anforderungen, die permanente Anspannung und das Problemverhalten der unterrichteten Schulkinder bei. Im Vergleich dazu wird das Unterrichten selbst in der Regel nicht als besonders belastend empfunden (Forneck \& Schriever, 2001).

Zusammen mit der Arbeitsbelastung bildet die Arbeits(un)zufriedenheit den Kernbereich der auf internationaler Ebene durchgeführten empirischen Untersuchungen zur beruflichen Gesamtsituation der Lehrpersonen. Gemäss dem Grundmodell der Berufszufriedenheit von Merz (1979) entsteht die Arbeits (un)zufriedenheit durch den Vergleich der objektiven Arbeitsanforderungen und -bedingungen mit den individuellen Erwartungen und Ansprüchen. Als Indikator für die Einstellung gegenüber der Berufstätigkeit ist die Arbeits(un)zufriedenheit häufig untersucht worden (z. B. Schmitz, 2000; Ipfling, Peez, \& Gamsjäger, 1995; Enzmann \& Kleiber, 1989; zusammenfassend, Bachmann, 1999; Rudow, 1994; Spear, Gould, \& Lee, 2000). Den Ergebnissen dieser internationalen Studien zufolge sind die meisten Lehrpersonen mit ihrem Beruf zufrieden; die Quelle dieser Zufriedenheit ist vor allem der Arbeitsinhalt (z.B. pädagogischer Entscheidungsspielraum, zwischenmenschliche Beziehungen). Als Gründe für Arbeitsunzufriedenheit werden u.a. Erziehungsprobleme und-misserfolge (z.B. Disziplinschwierigkeiten bei Schülern) genannt. Auch in der Schweiz gaben die meisten Lehrpersonen in Studien eine hohe allgemeine Arbeitszufrie- 
denheit an. Lehrpersonen finden ihren Beruf mehrheitlich attraktiv und sind gerne Lehrer bzw. Lehrerin (Bucher, 2001). Entsprechend bezeichneten in der Studie von Kramis-Aebischer (1996) knapp die Hälfte aller befragten Lehrpersonen ihre Berufszufriedenheit als hoch bis sehr hoch. 79\% der im Kanton Waadt befragten Lehrpersonen können sich in ihrer beruflichen Tätigkeit verwirklichen und $76 \%$ würden wieder denselben Beruf ergreifen, wenn sie nochmals vor der Wahl stünden (Gonik, Kurth, \& Boillat, 2000). Zu ähnlichen Ergebnissen kommt auch der Dachverband Schweizer Lehrerinnen und Lehrer: $71 \%$ der befragten Lehrpersonen würden nochmals den gleichen Beruf wählen (Landert, 2002). Im Vergleich zu einer Befragung aus dem Jahre 1990 nahm die Zustimmung allerdings ab. Nebst der hohen Bereitschaft, den Lehrberuf wieder zu ergreifen, zeigt die waadtländer Studie auch, dass immerhin $44 \%$ der befragten Lehrpersonen sich schon einmal überlegt hatten, aus dem Beruf auszusteigen (Gonik et al., 2000).

Nach Rudow (1994) führen die subjektiven Belastungen sowohl zu positiven als auch zu negativen psycho-physiologischen Beanspruchungsreaktionen. Während solche Reaktionen kurzfristig auftreten, können bei andauernder Beanspruchung chronische Folgen auftreten. Zu den möglichen positiven Beanspruchungsfolgen gehört u.a. Arbeitszufriedenheit. In Bezug auf negative Beanspruchungsfolgen, die zu einer Beeinträchtigung der Handlungskompetenz führen können, wurden vor allem chronischer Stress und Burnout untersucht (für Literaturüberblicke siehe u.a. Rudow, 1994; Barth, 1992; Kleiber \& Enzmann, 1990). Auch wiederholt aufgetretene oder chronische depressive und somatische Beschwerden gelten als Indikator für negative Beanspruchungsfolgen (Katholische Schule St. Alfons, 2002; Van Dick, 1999; Rudow, 1994). In der Schweiz konnte beispielsweise gezeigt werden, dass Stress und Erschöpfung unter Lehrpersonen im Kanton Waadt weit verbreitet sind und sich sogar derart verschlimmern können, dass der Beruf vorübergehend nicht ausgeübt werden kann (Masson, 1999). In der anderen waadtländer Studie von Gonik, Kurth und Boillat (2000) konnten die problematischen Arbeitsbedingungen mit der Ausprägung der somatischen und psychischen Symptome in Verbindung gebracht werden. Auch in den Kantonen Freiburg und Luzern bestätigt eine Untersuchung, dass Berufsbelastung und Arbeitsunzufriedenheit stark mit Depressivität und psychophysischen Beschwerden zusammenhängen (Kramis-Aebischer, 1996). Ferner ist die grosse psychische Belastung ein dominanter Kündigungsgrund bei Lehrpersonen (Bieri, 2003).

Eine umfassende Literatursuche zeigte, dass bisher keine Studie die Ausprägung von Arbeitsunzufriedenheit und von psychischer Belastung durch Arbeitsüberforderung bei Lehrpersonen in der Schweiz mittels international validierter Erhebungsinstrumente untersuchte. Die vorliegende Arbeit beschreibt daher erstmalig anhand einer national repräsentativen Stichprobe von Lehrpersonen der 5. bis 9. Klassen in der Schweiz die psychometrischen Qualitäten der beiden Skalen Arbeitsüberforderung und Arbeitsunzufriedenheit, welche von 
Enzmann und Kleiber (1989) zur Erfassung der empfundenen Belastung in der konkreten beruflichen Tätigkeit entwickelt wurden. Darüber hinaus beschreibt die Arbeit die Ausprägung von Depressivität und somatischen Beschwerden bei Lehrpersonen in den verschiedenen Sprachregionen und die Zusammenhänge mit Arbeitsüberforderung und -unzufriedenheit.

\section{Me thode}

\section{Studien- und Stichprobenbeschreibung}

Im Rahmen der Studie "Health Behaviour in School-Aged Children (HBSC)», die unter der Schirmherrschaft der Weltgesundheitsorganisation (WHO) in verschiedenen, zum grössten Teil europäischen Ländern alle vier Jahre durchgeführt wird, wird das Alltagsleben und die Gesundheit von Schulkindern im Alter zwischen 11 und 16 Jahren erhoben. Ziel dieser internationalen Studie ist es, Daten über das Gesundheitsverhalten der Jugendlichen und allfällige Veränderungen dieses Verhaltens im Laufe der Zeit zu sammeln. Im Jahre 2002 führte die Schweizerische Fachstelle für Alkohol- und andere Drogenprobleme (SFA) mit finanzieller Unterstützung des Bundesamtes für Gesundheit (BAG) und der Kantone diese Studie zum fünften Mal (nach 1986, 1990, 1994 und 1998) für die Schweiz durch.

Neben den Antworten der Schülerinnen und Schüler wurden auch die Ansichten der Lehrpersonen über die Gesundheitserziehung an der Schule sowie ihre eigene Situation hinsichtlich der Belastung durch ihre berufliche Tätigkeit und ihres Gesundheitsstatus mit einem separaten Lehrpersonenfragebogen erfasst. Wie den Schulkindern stand den Lehrpersonen eine Schulstunde zur Verfügung, um ihren Fragebogen auszufüllen. Ihre Teilnahme war ebenfalls freiwillig und die resultierenden Daten wurden anonym und streng vertraulich behandelt. Die Daten wurden zwischen März und Juni 2002 erhoben.

Die Zufallsauswahl der Klassen zwischen dem 5. und 9. Schuljahr und ihrer Lehrpersonen wurde anhand des Verzeichnis des Bundesamtes für Statistik (BFS), in dem alle Klassen der öffentlichen Schulen der Schweiz aufgelistet sind, vorgenommen. Im Jahre 2002 wurden 689 Klassen aus allen Sprachregionen der Schweiz zufällig ausgewählt. In den 589 Klassen, die an der Untersuchung teilnahmen, haben 577 Lehrpersonen geantwortet. Daraus ergibt sich eine Rücklaufquote von $83.7 \%$ bezogen auf die ausgewählten Klassen und von $98.0 \%$ bezogen auf die teilnehmenden Klassen. Die Auswahl kann als repräsentativ für die Lehrpersonen der 5. bis 9. Klassen in der gesamten Schweiz gelten. Weiterführende Informationen über die Lehrpersonenbefragung im Jahr 2002 in der Schweiz finden sich in Kuendig, Kuntsche, Delgrande Jordan und Schmid (2003).

Da das Erziehungsdepartement des Kantons Waadt bereits 1999 eine detaillierte Untersuchung zur Arbeitsbelastung und Gesundheit bei Lehrpersonen 
(Gonik et al., 2000) durchgeführt hatte, erhielten die waadtländer Lehrpersonen $(\mathrm{n}=44)$ eine kürzere Version des Fragebogens, in der u. a. die Fragen zur Arbeitsüberforderung und -unzufriedenheit sowie zu den Beschwerden nicht enthalten waren. Die waadtländer Lehrpersonen und andere, welche nicht alle der analysierten Fragen beantwortet hatten, wurden aus den Analysen ausgeschlossen $(n=92 ; 15.9 \%)$. Die resultierende Stichprobe enthält daher 485 Lehrpersonen. Es zeigt sich, dass in der Stichprobe die männlichen Lehrpersonen gegenüber den weiblichen in der Überzahl sind (Männer: 66.6\%; Frauen: 33.4\%) und dass das Durchschnittsalter bei 42.6 Jahren lag. Die Verteilung auf die Sprachregionen (Deutsch: 79.2\%; Französisch: 14.2\%; Italienisch: 6.6\%) unterstreicht die Repräsentativität der Stichprobe auf nationaler Ebene. Ferner ist jedes Schuljahr von der 5. bis zur 9. Klasse etwa gleich gut vertreten.

\section{Die Erhebungsinstrumente}

Die beiden Skalen Arbeitsüberforderung und Arbeitsunzufriedenheit wurden von Enzmann und Kleiber (1989) zur Erfassung der psychischen Belastung in der konkreten beruflichen Tätigkeit entwickelt. Die Autoren stützten sich bei der Itemauswahl auf einen bereits früher entwickelten Fragebogen in dem Modellversuch «Fachkräfte für die psychosoziale Versorgung» der Freien Universität Berlin und der FHSS-Berlin (Enzmann \& Kleiber, 1989). Das Erhebungsinstrument wurde in einem mehrsprachigen Forscherteam ins Französische und ins Italienische übersetzt, die Qualität der Übersetzung wurde somit in mehreren Diskussionen sichergestellt. Beide Skalen bestehen aus je sechs Items, ${ }^{2}$ die jeweils ein fünfstufiges Antwortformat haben $(1=$ trifft gar nicht zu, $2=$ trifft wenig zu, 3 = trifft mittelmäßig zu, $4=$ trifft überwiegend zu und $5=$ trifft völlig zu) und sich im schulischen Kontext als praktikable und zuverlässige Erhebungsinstrumente erwiesen haben (Schwarzer \& Jerusalem, 1999). Für die einfaktoriellen Varianzanalysen und die Regressionsanalysen wurden die 6 Items zur Arbeitsüberforderung und die 6 Items zur Arbeitsunzufriedenheit summiert (da die Items 1, 3, 4 und 5 in Richtung Arbeitszufriedenheit formuliert sind, wurden ihre Werte vorher invertiert codiert) und durch die Anzahl einbezogener Items dividiert.

Beschwerden. Die Batterie, die Items zu Beschwerden enthält, wurde eingeleitet mit «Wie oft hatten Sie in den letzten 6 Monaten die folgenden Beschwerden?» und mittels fünfstufigem Antwortformat erfasst $(5=$ etwa täglich, $4=$ mehrmals pro Woche, 3 = ungefähr einmal pro Woche, 2 = ungefähr einmal pro Monat und $1=$ selten oder nie). Die Beschwerden waren folgende: Kopfschmerzen, Bauchschmerzen, Rückenschmerzen, Schwindelanfälle, traurig/bedrückt, gereizt/schlecht gelaunt, nervös, müde und ängstlich/besorgt. Für die einfaktoriellen Varianzanalysen und die Regressionsanalysen wurden die 5 Items zur Depressivität und die 4 Items zu somatischen Beschwerden summiert und durch die Anzahl einbezogener Items dividiert. 


\section{Statistische Analysen}

Zur Bestimmung der Dimensionalität und psychometrischen Qualität zwölf arbeitsbezogener Variablen wurden varimax-rotatierte Hauptkomponentenanalysen und die internen Konsistenzen der resultierenden Faktoren (Cronbachs Alpha) berechnet. Auf die gleiche Weise wurde auch mit den 9 beschwerdebezogenen Variablen verfahren.

Da die Daten der vorliegenden Studie auf einer national repräsentativen Stichprobe beruhen, wurden Unterschiede in Arbeitsüberforderung, Arbeitsunzufriedenheit, Depressivität und somatischen Beschwerden in den drei Sprachregionen Deutsch, Französisch und Italienisch betrachtet. Resultierende Mittelwertsunterschiede wurden mittels einfaktorieller Varianzanalysen auf Signifikanz geprüft (F-Statistik). Ferner wurden Bonferroni Post-Hoc-Tests durchgeführt.

Zur Bestimmung der Zusammenhänge zwischen den arbeitsbezogenen Variablen Arbeitsüberforderung und -unzufriedenheit, Depressivität sowie somatischen Beschwerden wurden multiple Regressionsanalysen berechnet. Um zu überprüfen, ob sich diese Zusammenhänge in den Sprachregionen unterscheiden, wurden auf bivariater Ebene Korrelationen (Pearsons r) mittels Z-Tests (Kanji, 1999) auf Unterschiedlichkeit geprüft.

\section{Ergebnisse}

Die Hauptkomponentenanalyse der zwölf arbeitsbezogenen Variablen zeigt, dass sie klar in zwei zugrunde liegende Dimensionen bzw. Faktoren eingeteilt werden können (Tabelle 1). Mit «Cronbachs Alpha»-Werten über .75 haben beide Faktoren eine gute interne Konsistenz und erklären gemeinsam über die Hälfte der Varianz der einbezogenen Variablen. Auf dem ersten Faktor laden Items zur Arbeitsunzufriedenheit. Bis auf das Item «Ich habe mir schon ernsthaft überlegt, aus dem Beruf auszusteigen» zeigen sich hohe und homogene Ladungsmuster. Auf dem zweiten Faktor laden Items zur Arbeitsüberforderung. Aus dem sonst hohen und homogenen Ladungsmuster weichen die Items «Oft habe ich ein schlechtes Gewissen den Schülerinnen und Schülern gegenüber» und «Für andere Menschen verantwortlich zu sein, belastet mich sehr» etwas ab. Hier ist ausserdem festzuhalten, dass die zwei Dimensionen Arbeitsunzufriedenheit und Arbeitsüberforderung in starkem Zusammenhang stehen ( $\mathrm{r}=.47)$.

In der Tabelle 1 sind auch die Mittelwerte und Standardabweichungen der jeweiligen arbeitsbezogenen Variablen angeführt. Es zeigt sich, dass das Item zur Arbeitsunzufriedenheit «Ich habe mir schon ernsthaft überlegt, aus dem Beruf auszusteigen» die höchste Zustimmung hat, wohingegen das Item «Mein Beruf macht mir (keinen) Spass» die geringste Zustimmung aufweist. In Bezug auf die Arbeitsüberforderung ist das "Gefühl, nicht richtig abschalten zu können» am weitesten verbreitet, während die Items zum schlechten Gewissen gegenüber den Schulkindern und zur Verantwortung für andere Menschen die geringste Zustimmung aufweisen. 
Tab. 1: Varimax-rotatierte Hauptkomponentenanalyse arbeitsbezogener Variablen (Faktorenladungen, Mittelwerte, Standartabweichungen, Varianzaufklärung, Lamda-Werte und interne Konsistenz).

\begin{tabular}{|c|c|c|c|}
\hline & $\begin{array}{c}\text { Arbeits- } \\
\text { unzufriedenheit }\end{array}$ & $\begin{array}{c}\text { Arbeits- } \\
\text { überforderung }\end{array}$ & Mittelwert (SD) \\
\hline Mein Beruf macht mir (keinen)* Spass & .77 & & $1.90(.70)$ \\
\hline $\begin{array}{l}\text { Ich habe mir schon ernsthaft überlegt, } \\
\text { aus dem Beruf auszusteigen }\end{array}$ & .60 & & $2.48(1.37)$ \\
\hline $\begin{array}{l}\text { Was meine Arbeit betrifft, bin ich eigent- } \\
\text { lich rundum (nicht)* zufrieden }\end{array}$ & .71 & & $2.37(.85)$ \\
\hline $\begin{array}{l}\text { Ich halte mich (nicht)* gern an meinem } \\
\text { Arbeitsplatz auf }\end{array}$ & .66 & & $2.27(.93)$ \\
\hline $\begin{array}{l}\text { Ich kann in meinem Beruf meine Fähigkei- } \\
\text { ten (nicht)* voll nutzen }\end{array}$ & .75 & & $2.21(.91)$ \\
\hline $\begin{array}{l}\text { Meine eigenen Berufsideale lassen sich in } \\
\text { meiner Tätigkeit nicht verwirklichen }\end{array}$ & .64 & & $2.21(.99)$ \\
\hline $\begin{array}{l}\text { In meinem Beruf wird man ständig } \\
\text { überfordert }\end{array}$ & & .74 & $2.74(.90)$ \\
\hline Ich fühle mich häufig überfordert & & .77 & $2.12(.92)$ \\
\hline $\begin{array}{l}\text { Ich habe selten das Gefühl, einmal richtig } \\
\text { abschalten zu können }\end{array}$ & & .69 & $2.81(1.27)$ \\
\hline $\begin{array}{l}\text { Oft habe ich ein schlechtes Gewissen den } \\
\text { Schülerinnen und Schülern gegenüber }\end{array}$ & & .42 & $1.80(.74)$ \\
\hline $\begin{array}{l}\text { Für andere Menschen verantwortlich zu } \\
\text { sein, belastet mich sehr }\end{array}$ & & .55 & $1.93(.88)$ \\
\hline $\begin{array}{l}\text { Der Zeitdruck, unter dem ich arbeite, } \\
\text { ist zu gross }\end{array}$ & & .75 & $2.73(1.07)$ \\
\hline Varianzaufklärung & $25.34 \%$ & $24.82 \%$ & \\
\hline Lambda & 3.04 & 2.97 & \\
\hline Interne Konsistenz (Cronbach's $\alpha$ ) & .79 & .77 & \\
\hline
\end{tabular}

Anmerkung: Die Codierung der mit * gekennzeichneten Fragen wurde invertiert. Demzufolge wurde ihrer Wortlaut aus Verständlichkeitsgründen umformuliert. Der ursprüngliche Wortlaut dieser Fragen ist in Anmerkungen abgebildet; aus Darstellungsgründen wurden Faktorenladungen unter .40 nicht abgebildet.

Analog zu arbeitsbezogenen Variablen wurde auch mit den neun beschwerdebezogenen Variablen verfahren. Wie aus Tabelle 2 ersichtlich ist, ergeben sich erneut zwei Dimensionen bzw. Faktoren, die insgesamt wiederum über die Hälfte der Varianz der einbezogenen Items aufklären. Auf dem ersten Faktor laden beschwerdebezogene Variablen, die mit Depressivität in Verbindung stehen. Bis auf die Frage zur Müdigkeit zeigen sich hohe und homogene Ladungsmuster. Die interne Konsistenz der fünf Items zur Depressivität ist mit über .80 ausgezeichnet. Obwohl auch die Items zum zweiten Faktor recht hohe und homogene Ladungsmuster aufweisen, fällt die interne Konsistenz deutlich geringer aus. Es 
handelt sich also bei somatischen Beschwerden um ein weniger homogenes Syndrom als bei der Depressivität: Jemand, der zum Beispiel Rückenschmerzen oder Schwindelanfälle hat, hat eine nicht so grosse Wahrscheinlichkeit, ebenfalls unter den anderen somatischen Beschwerden zu leiden.

Ausserdem zeigt sich, dass Lehrpersonen im Durchschnitt am häufigsten von allen erfragten Beschwerden von Müdigkeit betroffen sind. Nervosität und schlechte Laune werden vergleichsweise seltener angegeben, kommen aber auch oft vor. Am wenigsten verbreitet sind Traurigkeit und Ängstlichkeit. Rückenschmerzen sind die häufigsten somatischen Beschwerden, Schwindelanfällen die seltensten.

Tab. 2: Varimax-rotatierte Hauptkomponentenanalyse beschwerdebezogener Variablen (Faktorenladungen, Mittelwerte, Standartabweichungen, Varianzaufklärung, Lamda-Werte und interne Konsistenz).

\begin{tabular}{|l|c|c|c|}
\hline & Depressivität & $\begin{array}{c}\text { somatische } \\
\text { Beschwerden }\end{array}$ & Mittelwert (SD) \\
\hline Traurig, bedrückt & .76 & & $1.81(.88)$ \\
Gereizt, schlecht gelaunt & .77 & & $2.22(.90)$ \\
Nervös & .81 & & $2.22(1.07)$ \\
Müde & .54 & & $2.96(1.06)$ \\
Ängstlich, besorgt & .79 & .76 & $1.80(.97)$ \\
Kopfschmerzen & & .68 & $1.61(.83)$ \\
Bauchschmerzen & & .53 & $1.33(.65)$ \\
Rückenschmerzen & & .61 & $1.94(1.17)$ \\
Schwindelanfälle & $31.4 \%$ & $21.2 \%$ & $1.21(.61)$ \\
\hline Varianzaufklärung & 2.83 & 1.91 & \\
Lambda & .81 & .54 & \\
Interne Konsistenz & & & \\
(Cronbach's $\alpha)$ & & &
\end{tabular}

Anmerkung: Aus Darstellungsgründen wurden Faktorenladungen unter .40 nicht abgebildet.

Für die weiteren Analysen wurden die zu den einzelnen Faktoren geladenen Variablen summiert und durch die Anzahl einbezogener Variablen pro Faktor geteilt. Aus Tabelle 3 ist ersichtlich, dass Arbeitsunzufriedenheit weniger verbreitet ist als Arbeitsüberforderung $(\mathrm{t}=3.6, \mathrm{p}<.001)$. Im Vergleich zur Arbeitsunzufriedenheit ist die Arbeitsüberforderung unterschiedlich auf die Sprachregionen verteilt. Bonferroni-Tests ergeben, dass sie in der Romandie häufiger vorkommt als in der Deutschschweiz $(\Delta \mu=.29, \mathrm{p}<.01)$. Im Bezug auf die Beschwerden zeigt sich, dass somatische Beschwerden weniger verbreitet sind als Depressivität $(t=21.25, \mathrm{p}<.001)$. Im Gegensatz zu den somatischen Beschwerden ergibt sich 
für Depressivität ein sprachregionaler Unterschied. Bonferroni-Tests belegen, dass Depressivität in der Romandie häufiger vorkommt als in der Deutschschweiz $(\Delta \mu=.38, \mathrm{p}<.001)$.

Tab. 3: Mittelwerte arbeitsbezogener Variablen und Beschwerden gesamt und nach Sprachregion (Standartabweichungen in Klammern und F-Werte der einfaktoriellen Varianzanalyse).

\begin{tabular}{|l|c|c|c|c|c|}
\hline & Total & Deutsch & Französisch & Italienisch & F \\
\hline Arbeitsbezogenen Variablen & & & & & \\
Arbeitsunzufriedenheit & $2.24(.69)$ & $2.22(.66)$ & $2.30(.76)$ & $2.35(.83)$ & 0.9 n.s. \\
Arbeitsüberforderung & $2.37(.66)$ & $2.30(.64)$ & $2.59(.74)$ & $2.51(.68)$ & $6.7^{* *}$ \\
Beschwerden & & & & & \\
Depressivität & $2.20(.74)$ & $2.13(.69)$ & $2.50(.91)$ & $2.39(.79)$ & $8.9^{* * *}$ \\
Somatische Beschwerden & $1.52(.55)$ & $1.50(.54)$ & $1.54(.44)$ & $1.71(.77)$ & 2.2 n.s. \\
\hline
\end{tabular}

Anmerkungen: ${ }^{* *} \mathrm{p}<0.01 ;{ }^{* * *} \mathrm{p}<0.001$.

Ferner wurden die Arbeits- und die Beschwerdeskalen miteinander in Beziehung gesetzt. Tabelle 4 enthält die Ergebnisse multipler Regressionsanalysen zur Bestimmung von Depressivität und somatischen Beschwerden. In beiden Fällen weist die Arbeitsüberforderung einen höheren Zusammenhang auf als Arbeitsunzufriedenheit, jedoch sind alle vier Koeffizienten auf einem hohen Niveau statistisch signifikant. Knapp die Hälfte der Varianz von Depressivität konnte durch Arbeitsüberforderung und -unzufriedenheit erklärt werden. Im Fall von somatischen Beschwerden waren es 12 Prozent.

Tab. 4: Determinanten von Depressivität und somatischen Beschwerden von Lehrpersonen (standardisierte Regressionskoeffizienten Beta, t-Werte in Klammern und Varianzaufklärung).

\begin{tabular}{|l|c|c|}
\hline & Depressivität & $\begin{array}{c}\text { somatische } \\
\text { Beschwerden }\end{array}$ \\
\hline Arbeitsunzufriedenheit & $.22^{* * *}(5.68)$ & $.13^{* *}(2.69)$ \\
Arbeitsüberforderung & $.52^{* * *}(13.32)$ & $.27^{* * *}(5.47)$ \\
\hline Varianzaufklärung $\left(\mathrm{R}^{2}\right)$ & $43.2 \%$ & $12.0 \%$ \\
\hline
\end{tabular}

Anmerkung: Alle Analysen sind für Geschlecht und Alter kontrolliert; ${ }^{* *} \mathrm{p}<0.01 ;{ }^{* * *} \mathrm{p}<0.001$.

Die in Tabelle 4 dargestellten Ergebnisse wurden zusätzlich auf bivariater Ebene mittels Korrelationen (Pearsons r) für die drei Sprachregionen getrennt nachgerechnet und die Zusammenhänge mittels Z-Tests auf Unterschiedlichkeit geprüft. Keine der gefundenen Differenzen erreichte jedoch statistische Signifi- 
kanz. Dies bedeutet, dass die dargestellten Zusammenhänge für alle drei Sprachregionen Bedeutung besitzen.

\section{Zusammenfassung und Diskussion}

Die vorliegende Arbeit hatte die Aufgabe, die psychometrischen Qualitäten der beiden Skalen Arbeitsüberforderung und Arbeitsunzufriedenheit (Enzmann \& Kleiber, 1989; Schwarzer \& Jerusalem, 1999) anhand einer national repräsentativen Stichprobe von Lehrpersonen der 5. bis 9. Klassen in der Schweiz zu erfassen. In Übereinstimmung mit vorangegangener Forschung konnten in einer Hauptkomponentenanalyse Arbeitsüberforderung und -unzufriedenheit als separate Dimensionen identifiziert werden (Enzmann \& Kleiber, 1989; Schwarzer \& Jerusalem, 1999). Zudem bestätigen «Cronbachs Alpha»-Werte von über .75 die gute interne Konsistenz der beiden Skalen.

Insgesamt zeigt sich, dass Lehrpersonen in der Schweiz im Durchschnitt wenig bis mittelmässig von Arbeitsüberforderung betroffen sind. Allerdings muss dieser Befund nuanciert werden, denn eine nicht geringe Anzahl von Lehrpersonen gab an, vom Gefühl, nicht richtig abschalten zu können, und unter Zeitdruck arbeiten zu müssen. Diese Ergebnisse passen weitgehend zu denjenigen von vorangegangenen Arbeiten in der Schweiz (Ulich et al. , 2002; KramisAebischer, 1996; Frei, 1996). Der Vergleich ist jedoch schwierig, weil die psychische Belastung in den anderen Studien zu diesem Thema jeweils anders operationalisiert wurde. Hinsichtlich der Arbeitsunzufriedenheit fällt zunächst auf, dass sie seltener vorkommt als die Arbeitsüberforderung. Insgesamt scheint es, dass wenige Lehrpersonen mit ihrem Beruf unzufrieden sind, was wiederum mit früheren schweizbezogenen Ergebnissen übereinstimmt (Bucher, 2001; KramisAebischer, 1996). Trotzdem gaben eine nicht zu vernachlässigende Anzahl von Lehrpersonen an, dass sie sich schon ernsthaft überlegt hatten, aus dem Beruf auszusteigen. Dies kann als weiterer Indikator für psychische Belastung angesehen werden.

Analog zu den arbeitsbezogenen Variablen wurde auch mit den beschwerdebezogenen Variablen verfahren. Hierbei konnten die Dimensionen «Depressivität» und «somatische Beschwerden» extrahiert werden. Im Falle von Depressivität liegt die interne Konsistenz sogar über .80. Dass die interne Konsistenz bei den somatischen Beschwerden geringer ausfällt, liegt daran, dass die Kumulation von Kopf-, Bauch- und Rückenschmerzen und Schwindelanfällen eher selten ist. Ausserdem fällt auf, dass mit Ausnahme der Rückenschmerzen Depressivitätssymptome öfter vorkommen als somatische Beschwerden; eine nicht geringe Gruppe von Lehrpersonen ist häufig müde, traurig, gereizt, nervös oder ängstlich. Auch andere Studien zeigten, dass Lehrpersonen zahlreiche somatische und psychische Symptome aufweisen (Gonik et al. , 2000; Kramis-Aebischer, 1996).

Im Vergleich der Sprachregionen zeigte sich, dass Arbeitsüberforderung und 
Depressivität in der Romandie häufiger vorkommen als in der Deutschschweiz. Hingegen konnten in Bezug auf Arbeitsunzufriedenheit und somatische Beschwerden keine sprachregionalen Unterschiede festgestellt werden. Mit anderen Worten sind die westschweizer Lehrpersonen mehr überfordert aber nicht unzufriedener als die deutschschweizer Lehrpersonen. Das mag vor allem an strukturellen Unterschieden liegen, z. B. an den erheblichen interkantonalen bzw. interregionalen Unterschieden in den Schulsystemen und in der Organisation des Unterrichts.

Im Einklang mit anderen Studien (vgl. Frei, 1996; Ulich et al. , 2002) konnten hier Arbeitsüberforderung und -unzufriedenheit mit der Ausprägung von Beschwerden in Verbindung gebracht werden: Je mehr Überforderung und Unzufriedenheit, desto mehr äussern die Lehrpersonen Beschwerden. Die multiplen Regressionsanalysen zeigen ausserdem, dass Arbeitsüberforderung und -unzufriedenheit, obwohl beide Dimensionen einen starken Zusammenhang aufweisen ( $\mathrm{r}=.47)$, einen additiven Beitrag zur Erklärung von Depressivität und somatischen Beschwerden liefern. Mit anderen Worten: Selbst bei einem bestehenden Ausmass an Arbeitsüberfoderung erhöht eine hohe Arbeitsunzufriedenheit die Wahrscheinlichkeit, unter Depressivität und somatischen Beschwerden zu leiden. Entsprechendes ist auch bei einem bestimmten Niveau an Arbeitsunzufriedenheit der Fall. Die Ergebnisse zeigen also, dass Arbeitsüberforderung und -unzufriedenheit unabhängig voneinander zur Erklärung von Depressivität und somatischen Beschwerden beitragen, obwohl arbeitsüberforderte Lehrpersonen häufig auch arbeitsunzufrieden sind (und umgekehrt). Fast die Hälfte der Gesamtvarianz von Depressivität konnte durch diese Dimensionen erklärt werden; ebenso zeigt sich, dass Arbeitsüberforderung und -unzufriedenheit auch mit somatischen Beschwerden einhergehen.

Die Ergebnisse unserer Studie sind Besorgnis erregend, da häufige somatische oder psychische Beschwerden bei überforderten Lehrpersonen ein Indikator für negative Beanspruchungsfolgen wie z. B. chronischen Stress oder Burnout sein können. Diese können gravierende Konsequenzen haben, u. a. eine Beeinträchtigung der pädagogischen Handlungskompetenz sowie psychische oder somatische Gesundheitsstörungen (Grunder \& Bieri, 1995).

Die vorliegende Studie basiert jedoch lediglich auf Querschnitt-Daten. Bei den beschriebenen Zusammenhängen handelt es sich nicht um kausale Effekte im Sinne einer Ursache-Wirkungsbeziehung. Es ist daher ebenfalls denkbar, dass depressive oder durch somatische Beschwerden beeinträchtigte Lehrpersonen eher anfällig für Arbeitsüberforderung und -unzufriedenheit sind (Grunder \& Bieri, 1995). Zudem war es auf der Basis der vorliegenden Daten nicht möglich, die Arbeitsüberforderung und -unzufriedenheit bei den Lehrpersonen mit anderen Berufsgruppen oder der Gesamtbevölkerung zu vergleichen. Auch die erheblichen interkantonalen Unterschiede in den Bildungssystemen als mögliche Erklärung für spachregionale Unterschiede konnten nicht berücksichtigt werden. Die Klärung dieser Fragestellungen bleibt somit Aufgabe zukünftiger Forschung. 


\section{Ausblick zu Prävention und Intervention}

Die Ergebnisse der vorliegende Studie zeigen, dass es weiterhin und insbesondere in der französisch- und italienischsprachigen Schweiz nötig ist, präventive und gesundheitsfördernde Massnahmen für Lehrpersonen zu ergreifen, und dass es notwendig ist, sowohl Arbeitsüberforderung als auch Arbeitsunzufriedenheit in den Blickpunkt präventiver Bemühungen zu rücken. Neben der Förderung individueller Ressourcen sind Verbesserungen auf strukturell-institutioneller Ebene wichtig; sie tragen zu einer positiven Veränderung des Schulklimas bei.

Auf der individuellen Ebene können präventive Massnahmen, die auf ein erfolgreiches Stressmanagement abzielen, eine wichtige Unterstützung für Lehrpersonen sein. Entspannungsübungen wie Autogenes Training oder die Progressive Muskelrelaxion nach Jacobson verbessern die Verhaltenskontrolle und regulieren negative Gefühle wie Angst oder Wut (Freitag, 1998). Auch Programme zum Erlernen des Umgangs mit Stress wie der Ansatz Selbstmanagement (Fontana, 1991) oder das psychologische Programm zur Gesundheitsförderung "Gelassen und sicher im Stress» (Kaluza, 1996) sind wichtige präventive Massnahmen auf der Verhaltensebene. Aufgrund der präsentierten Ergebnisse, die zeigen, dass eine nicht zu vernachlässigende Gruppe von Lehrpersonen angibt, ängstlich oder besorgt zu sein, sind auch Programme zur Angstbewältigung zu beachten. Solche Trainingsprogramme sollen Lehrpersonen befähigen, mit Angst auslösenden Situationen umzugehen.

Interventionen zu gesundheits- und persönlichkeitsfördernden Verhaltensänderung von Lehrpersonen sind nicht ohne die Entwicklung der Organisation Schule möglich (Freitag, 1998). Auf der strukturell-institutionellen Ebene müssen Arbeitsbedingungen für Lehrpersonen wie Klassengrösse, Lärm oder Lichtverhältnisse fokussiert und angepasst werden. Bei der Gestaltung der Arbeitsbedingungen kommt ausserdem der sozialen Unterstützung eine wichtige Bedeutung zu. Der Austausch mit Kollegen und Kolleginnen, die mit denselben Schwierigkeiten konfrontiert sind, ist häufig eine wichtige emotionale Unterstützung; er kann institutionell verankert werden. Auch die Reflexion von schwierigen Situationen kann Lehrpersonen bei der Entwicklung von Handlungskompetenzen helfen. Soziale Unterstützungssysteme für Lehrpersonen wie Selbsthilfegruppen oder kollegiale Supervisionen sollten integraler Bestandteil schulorganisatorischer Massnahmen sein (Freitag, 1998).

Weitere Massnahmen zur Vermeidung von Arbeitsüberforderung bei Lehrpersonen sind auch in der Aus- und Weiterbildung zu verankern. Lattmann und Rüedi (2003) geben zu bedenken, dass die bisher diskutierte Belastungsperspektive durch die Ressourcenperspektive ergänzt werden muss. Durch die Stärkung protektiver Faktoren und von Bewältigungsressourcen wie etwa einem positiven Selbstwertgefühl wird ein anderer Umgang mit den Anforderungen gelernt (Freitag, 1998). Das Projekt «Stress- und Selbstmanagement in der Schule» soll bei Lehrpersonen Ressourcen und Kompetenzen fördern, die eine positive und 
konstruktive Gestaltung von Belastungssituationen im Beruf ermöglichen (Lattmann, 2003). Die Lehrpersonen erwerben verschiedene Techniken, die ihnen ermöglichen, sich und andere besser zu verstehen.

Eine gute und gesunde Schule (Paulus, 2003) nimmt auch die Gesundheit der Lehrpersonen als Herausforderung wahr und ergreift entsprechende Massnahmen auf verschiedenen Ebenen. Damit kann nicht nur der Arbeitsüberforderung, der Arbeitunzufriedenheit, der Depressivität und den somatischen Beschwerden vorgebeugt werden, sondern die präventiven Bemühungen tragen auch zu einem guten Schulklima und damit zur guten und gesunden Schule bei.

\section{Anmerkungen}

1 Die Lehrpersonenstudie wurde im Rahmen der Studie «Health Behaviour in School-Aged Children (HBSC)» durchgeführt (Bundesamt für Gesundheit, Projektnummer 00.000300), Projektleitung: Dr. H. Schmid. Finanziert wurde die Eingabe und Auswertung der Lehrpersonenstudie durch SFA Eigenmittel. Der vorliegende Artikel basiert teilweise auf einem Vortrag, der an der nationalen Impulstagung des Schweizerischen Netzwerks gesundheitsfördernder Schulen am 22. November 2003 in Luzern gehalten wurde.

2 Die Items lauten im Originalwortlaut (Enzmann \& Kleiber, 1989) für die Skala Arbeitsunzufriedenheit: "Mein Beruf macht mir Spass», "Ich habe mir schon ernsthaft überlegt, aus dem Beruf auszusteigen", "Was meine Arbeit betrifft, bin ich eigentlich rundum zufrieden", "Ich halte mich gern an meinem Arbeitsplatz auf», "Ich kann in meinem Beruf meine Fähigkeiten voll nutzen» und «Meine eigenen Berufsideale lassen sich in meiner Tätigkeit nicht verwirklichen»; für die Skala Arbeitsüberforderung: «In meinem Beruf wird man ständig überfordert», "Ich fühle mich häufig überfordert», "Ich habe selten das Gefühl, einmal richtig abschalten zu können», "Oft habe ich ein schlechtes Gewissen den Schülerinnen und Schülern gegenüber», «Für andere Menschen verantwortlich zu sein, belastet mich sehr» und «Der Zeitdruck, unter dem ich arbeite, ist zu gross».

\section{Literatur}

Bachmann, K. (1999). Lust oder Last - Berufszufriedenheit und Belastung im Beruf bei Lehrerinnen und Lehrern an berufsbildenden Schulen. Baltmannsweiler: Schneider Verlag Hohengehren $\mathrm{GmbH}$.

Barth, A.-R. (1992). Burnout bei Lehrern. Theoretische Aspekte und Ergebnisse einer Untersuchung. Göttingen: Hogrefe.

Bieri, T. (2003). Die berufliche Situation in der Perspektive der Lehrerinnen und Lehrer. Bern: Universität Bern.

Bucher, B. (2001). Arbeitszeit und Arbeitsbelastung der Lehrpersonen - Einsichten, Aussichten, Folgerungen (Literaturbericht). Luzern: Bildungsplanung Zentralschweiz.

Enzmann, D., \& Kleiber, D. (1989). Helfer-Leiden: Stress und Burnout in psychosozialen Berufen. Heidelberg: Asanger.

Fontana, D. (1991). Mit dem Stress leben. Bern: Verlag Hans Huber.

Forneck, H. J., \& Schriever, F. (2001). Die individualsisierte Profession: Belastungen im Lehrberuf. Bern: H.e.p.- Verlag.

Frei, B. (1996). Belastungen im Lehrberuf. Zürich: Pädagogisches Institut der Universität Zürich.

Freitag, M. (1998). Was ist eine gesunde Schule? Einflüsse des Schulklimas auf Schüler-und Lehrergesundheit. Weinheim / München: Juventa Verlag. 
Gonik, V., Kurth, S., \& Boillat, M.-A. (2000). Analyse du questionnaire sur l'état de santé physique et mentale des enseignants vaudois (Rapport final). Lausanne: Institut universitaire romand de Santé au Travail (IST).

Grunder, H.-U., \& Bieri, T. (1995). Zufrieden in der Schule - zufrieden mit der Schule? Bern: Haupt.

Ipfling, H. J., Peez, H., \& Gamsjäger, E. (1995). Wie zufrieden sind die Lehrer? Bad Heilbrunn/Obb.: Klinkhardt.

Jarvis, M. (2002). Teacher stress: A critical review of recent findings and suggestions for future research directions. Stress News, 14 (1), http://www.isma.org.uk/stressnw/teachstress 1.htm [6.2.2005].

Kaluza, G. (1996). Gelassen und sicher im Stress. Psychologisches Programm zur Gesundheitsförderung. Heidelberg: Springer.

Kanji, G. K. (1999). 100 statistical tests. London: Sage.

Katholische Schule St. Alfons. (2002). Lehrer werden krank durch die Schule. http://www.kssa.delindex-Dateien/page227.html [11.02.2004].

Kleiber, D., \& Enzmann, D. (1990). Burnout. Eine internationale Bibliographie - An International Bibliography. Göttingen: Verlag für Psychologie Dr. C.J. Hogrefe.

Kramis-Aebischer, K. (1996). Stress, Belastungen und Belastungsverarbeitung im Lehrberuf (2., unveränderte Auflage). Bern / Stuttgart / Wien: Haupt.

Kuendig, H., Kuntsche, E. N., Delgrande Jordan, M., \& Schmid, H. (2003). Gesundheitserziehung in der Schule: Standpunkte der Lehrkräfte - Deskriptive Statistik der 2002 erhobenen Daten. Lausanne: Schweizerische Fachstelle für Alkohol- und andere Drogenprobleme.

Landert, C. (2002). LCH-Umfrage Berufszufriendenheit: Ständige Reformen machen Lehrpersonen zu schaffen. Bildung Schweiz, 10, 15-19.

Lattmann, U. P. (2003). Stress- und Selbstmanagement als Ressourcenförderung: Das Projekt Strema. In U. P. Lattmann \& J. Rüedi (Hrsg.), Stress- und Selbstmanagement-Ressourcen födern. Konzept und Programme zur Förderung personaler und sozial-kommunikativer Ressourcen und Kompetenzen (S. 73-112). Aarau: Sauerländer Verlag.

Lattmann, U. P., \& Rüedi, J. (2003). Gesundheit und Schule. In U. P. Lattmann \& J. Rüedi (Hrsg.), Stress- und Selbstmanagement - Ressourcen fördern. Konzept und Programme zur Förderung personaler und sozial-kommunikativer Ressourcen und Kompetenzen. (S. 45-72). Aarau: Sauerländer Verlag.

Masson, S. (1999). La pénibilité du travail des maîtres secondaires. Lausanne: Société Vaudoise des Maitres Secondaires (SVMS).

Merz, J. (1979). Berufszufriedenheit von Lehrern. Eine empirische Untersuchung. Weinheim I Basel: Beltz.

Paulus, P. (2003). Schulische Gesundheitsförderung - vom Kopf auf die Füsse gestellt. Von der Gesundheitsfördernden Schule zur guten, gesunden Schule. In K. Aregger \& U. P. Lattmann (Hrsg.), Gesundheitsfördernde Schule - eine Utopie? Konzepte, Praxisbeispiele, Perspektiven. Aarau: Sauerländer Verlag.

Rudow, B. (1994). Die Arbeit des Lehrers. Zur Psychologie der Lehrertätigkeit, Lehrer-belastung und Lehrergesundheit. Bern: Hans Huber.

Schaarschmidt, U., \& Fischer, A. (2001). Bewältigungsmuster im Beruf. Persönlichkeitsunterschiede in der Auseinandersetzung mit der Arbeitsbelastung. Göttingen: Vandenhoeck \& Ruprecht.

Schmitz, G. S. (2000). Zur Struktur und Dynamik der Selbstwirksamkeitserwartung von Lehrern. Ein protektiver Faktor gegen Belastung und Burnout? Digitale Dissertation. http://www.diss.fu-berlin.de/2000/29/index.html [3.11.2001].

Schwarzer, R., \& Jerusalem, M. (Hrsg.). (1999). Skalen zur Erfassung von Lehrer- und Schülermerkmalen. Dokumentation der psychometrischen Verfahren im Rahmen der Wissenschaftlichen Begleitung des Modellversuchs Selbstwirksame Schulen. Berlin: Freie Universität Berlin. 
Spear, M., Gould, K., \& Lee, B. (2000). Who would be a teacher? A review of factors motivating and demotivating prospective and practising teachers. Slough: NFER.

Ulich, E., Inversini, S., \& Wülser, M. (2002). Arbeitsbedingungen, Belastungen und Ressourcen der Lehrkräfte des Kantons Basel-Stadt. Zürich: Institut für Arbeitsforschung und Organisationsberatung.

Van Dick, R. (1999). Stress und Arbeitszufriendenheit im Lehrberuf (Wissenschaftliche Beiträge, Reihe Psychologie. Band 2). Marburg: Tectum Verlag.

Schlagworte: Arbeitszufriedenheit, Berufsbelastung, Lehrpersonen, Beschwerden, Prävention

\section{Surcharge de travail et insatisfaction professionnelle chez les enseignant-e-s en Suisse - liens avec la dépressivité et les symptômes somatiques}

\section{Resumé}

Cette contribution décrit pour la première fois sur la base d'un échantillon d'enseignant-e-s de 5e à 9e années scolaires représentatif pour la Suisse les qualités psychométriques des échelles «surcharge de travail» et «insatisfaction professionnelle» de Enzmann et Kleiber (1989) ainsi que leur lien avec la dépressivité et les symptômes somatiques. Les réponses de 485 enseignant-e-s y sont analysées au moyen d'analyses en composantes principales et de régressions multiples. En accord avec les résultats d'autres études, la surcharge de travail et l'insatisfaction professionnelle ont été identifiées comme des dimensions séparées. La surcharge de travail et la dépressivité sont plus répandues en Suisse romande qu'en Suisse alémanique. En revanche, aucune différence entre régions linguistiques n'a été trouvée en ce qui concerne l'insatisfaction professionnelle et les symptômes somatiques. Plus d'un tiers de la variance totale de la dépressivité peut ainsi être expliquée uniquement par la surcharge de travail et l'insatisfaction professionnelle. Ces dernières sont en outre associées à des symptômes somatiques. Plus les enseignant-e-s sont surchargé-e-s et insatisfait-e-s, plus ils/elles déclarent souffrir de troubles somatiques ou psychiques. Ce constat est préoccupant dans la mesure où les symptômes somatiques et psychiques chez les enseignant-e-s surchargé-e-s peuvent être un indicateur de stress chronique ou de burnout. Les mesures préventives présentées peuvent contribuer dans une certaine mesure à l'amélioration des conditions de travail des enseignant-e-s et du climat de travail dans les écoles.

Mots clés: satisfaction professionnelle, charge de travail, enseignants, prévention 


\title{
Sovraccarico di lavoro e insoddisfazione professionale fra gli insegnanti svizzeri - legami con la depressione e con sintomi somatici
}

\section{Riassunto}

Il contributo descrive per la prima volta e sulla base di un campione rappresentativo per gli insegnanti dal quinto al nono anno scolastico le caratteristiche psicometriche della scala "sovraccarico di lavoro" e "insoddisfazione professionale" di Enzmann e Kleiber (1989) e i relativi legami con la depressione e i sintomi somatici. Le risposte di 485 insegnanti sono state analizzate tramite segmentazione in componenti e regressione multipla. In accordo con altri studi del genere, il sovraccarico di lavoro e l'insoddisfazione professionale sono stati identificati separatamente. Si constata che il sovraccarico di lavoro e la depressione sono più diffuse in Svizzera romanda che in quella tedesca. Per contro non ci sono differenze regionali per gli altri due aspetti considerati. Di riflesso più di un terzo della varianza totale della depressione può essere spiegata attraverso il sovraccarico e l'insoddisfazione professionale, vale a dire più gli insegnanti sono sovraccarichi e insoddisfatti, più dichiarano di soffrire di sintomi somatici o psichici. Questa constatazione è preoccupante nella misura in cui questi sintomi possono denotare stress cronico e burnout. Vengono infine proposte delle misure preventive atte a contribuire al miglioramento delle condizioni di lavoro degli insegnanti e del clima nelle scuole.

Parole chiave: Soddisfazione professionale, sovraccarico professionale, disturbi, prevenzione

\section{Professional overload and dissatisfaction among teachers in Switzerland - associations with depressiveness and somatic complaints}

\begin{abstract}
Based on a representative sample for Switzerland, this paper describes, for the first time, the psychometric qualities of the scales "professional overload" and "professional dissatisfaction" from Enzmann and Kleiber (1989) as well as their association with depressiveness and somatic complaints among teachers of 5 th to 9 th grades. The answers of 485 teachers were analyzed using principal component analysis and multiple regressions. In accordance with the results of previous studies, "professional overload" and "professional dissatisfaction" are two different dimensions. Professional overload and depressiveness are more frequent in the French speaking part than in the German speaking part. On the other hand, no difference was found between the linguistic regions with regards to professional dissatisfaction and somatic complaints. More than one third of the vari-
\end{abstract}


ance of depressiveness is explained only by the two variables, professional overload and professional dissatisfaction. In addition, both variables are associated with somatic complaints. The more the teachers are overloaded and dissatisfied, the more they report somatic or psychological problems. This is alarming because these complaints may be an indicator of chronic stress or burnout. Preventive actions that can contribute to improve the work conditions of teachers are suggested and described at the end of the paper.

Key words: professional satisfaction, work stress, teachers, prevention 
